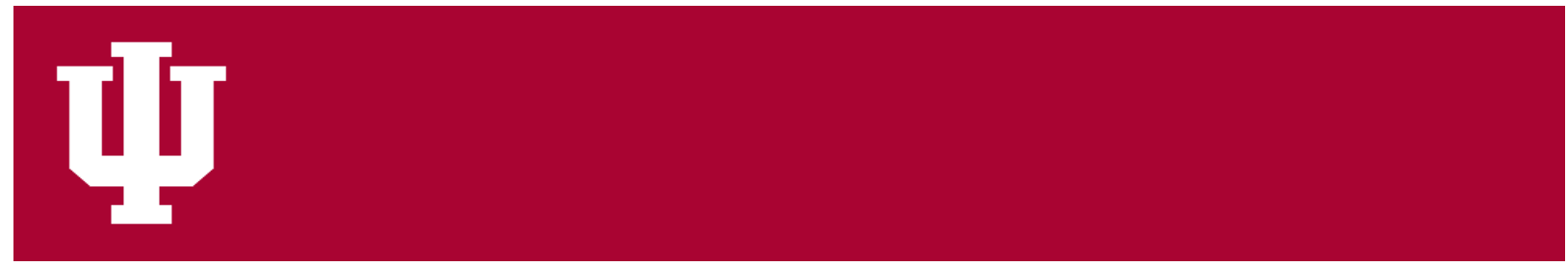

IUScholarWorks at Indiana University South Bend

\title{
Evidence for Nonequilibrium Proton Emission in a Low-Energy Heavy-Ion Reaction
}

DeYoung, P. A., Goupell, M. J., Atallah, B. V., Haglund, J. A., Jolivette, P. L., MacDermaid, M. K., Peaslee, G. F., Kolata, J. J., Berners, E. D., Peterson, D., von Schwarzenberg, J., \& Hinnefeld, J. D.

To cite this article: DeYoung, P. A., et al. "Evidence for Nonequilibrium Proton Emission in a Low-Energy Heavy-Ion Reaction." Physical Review C, vol. 61, no. 2, American Physical Society, Dec. 1999, p. 024603. APS, doi:10.1103/PhysRevC.61.024603.

This document has been made available through IUScholarWorks repository, a service of the Indiana University Libraries. Copyrights on documents in IUScholarWorks are held by their respective rights holder(s). Contact iusw@indiana.edu for more information. 


\title{
Evidence for nonequilibrium proton emission in a low-energy heavy-ion reaction
}

\author{
P. A. DeYoung, M. J. Goupell, B. V. Atallah, J. A. Haglund, P. L. Jolivette, M. K. MacDermaid, and G. F. Peaslee \\ Department of Physics, Hope College, Holland, Michigan 49422-9000
}

\author{
J. J. Kolata, E. D. Berners, D. Peterson, and J. von Schwarzenberg \\ Physics Department, University of Notre Dame, Notre Dame, Indiana 46556 \\ J. D. Hinnefeld \\ Physics Department, Indiana University-South Bend, South Bend, Indiana 46615
}

(Received 10 May 1999; published 30 December 1999)

\begin{abstract}
Correlation functions for $p-p, p-d, p-\alpha, d-\alpha$, and $\alpha-\alpha$ were measured for the ${ }^{16} \mathrm{O}+{ }^{27} \mathrm{Al}$ system at a beam energy of $72 \mathrm{MeV}$. The detector array was centered at laboratory angle of $20^{\circ}$. Compared to published data obtained at larger scattering angles, an unexpected dependence on angle is seen for the $p$ - $p$ channel. The observed anticorrelation is stronger at more forward angles, which suggests that correlation functions are sensitive to the specific reaction mechanisms producing the light-charged particles.

PACS number(s): 25.70.Pq, 25.70.Gh
\end{abstract}

\section{INTRODUCTION}

A large number of previous studies have been made of small-angle light-charged-particle (LCP) correlation functions for a variety of systems and energies [1]. A survey of these results revealed a wide variety of results and a dependence of the measured correlation functions on scattering angle [1]. In many previous reports, where source characteristics were inferred from correlation measurements, there is a tacit assumption that measurements spanning a small range of scattering angles would represent the overall behavior. The finding that the correlation functions vary with angle suggests that care must be taken when interpreting such measurements. It seems that different sources of LCP's contribute in differing ways to produce any particular correlation function.

The specific purpose of the measurement reported here was to observe the behavior of a low-energy system. These low-energy systems are generally well understood within the context of the statistical model allowing one to better understand the correlation measurements at higher energies. The specific system studied was ${ }^{16} \mathrm{O}+{ }^{27} \mathrm{Al}$ at $72 \mathrm{MeV}$. The particular model chosen to simulate the data includes only statistical emission from an equilibrated compound nucleus with no other nonstatistical emissions. In a similar correlation study at $80 \mathrm{MeV}$ [2], this assumption served well and is assumed to be valid here. This experiment was performed with the expectation that there would be no angular dependance observed because of the low energy of the reaction. However, an angular dependance was again observed.

\section{EXPERIMENTAL PROCEDURE AND DATA ANALYSIS}

This experiment was performed at the University of Notre Dame Nuclear Structure Laboratory. A $72 \mathrm{MeV}{ }^{16} \mathrm{O}$ beam was produced and impacted a thin ${ }^{27} \mathrm{Al}$ target $\left(700 \mu \mathrm{g} / \mathrm{cm}^{2}\right)$. Fourteen CsI detectors with a $1.5 \mathrm{~cm}$ diameter were placed in a hexagonal close packed array, $50 \mathrm{~cm}$ away from the target. The center angle of the array was $20.0^{\circ}$ with respect to the beam, while the angle between adjacent detectors was $3.27^{\circ}$. The most forward detector was at an angle of $16.9^{\circ}$.

The ${ }^{16} \mathrm{O}$ beam was pulsed and bunched in this measurement. The time resolution of the beam pulses was $1.5 \mathrm{~ns}$ and the pulse period was $100 \mathrm{~ns}$. An electrostatic beam sweeper system insured that the number of beam particles incident on the target between the beam bunches was negligible. The flight path from target to detector and the width of the beam pulse was such that individual masses could not be well resolved from the energy versus time-of-flight (TOF) data. However, the TOF data did allow a thorough measurement of the number of random coincidences between two LCP. During the entire course of the experiment the real to random ratio was greater than 100 to 1 .

Pulse shaping techniques were used for particle identification of protons, deuterons, tritons, and $\alpha$ particles [3]. There were slow gain variations, typically less than $5 \%$ /day, over the course of the week-long experiment. The shifts were measured by monitoring the scattered protons from the ${ }^{1} \mathrm{H}\left({ }^{16} \mathrm{O},{ }^{1} \mathrm{H}\right)$ reaction (with absorbed hydrogen in the aluminum target) and elastically scattered ${ }^{16} \mathrm{O}$. The energy calibration constants were then varied for each run to account for the shift. A similar correction was applied so that the particle identification was optimized from run to run. Resolution of the LCP energies is $1 \%$ (slightly larger at the very lowest energies). The final energy calibrations were determined from elastic scattering of protons and $\alpha$ particles off gold over a range of energies. Count rates in the detectors, including elastic scattered beam particles, were held to less than 3 $\mathrm{kHz}$ to minimize acquisition deadtime and enhance detector performance.

Coincident data were analyzed for events involving pairs of detectors with opening angles of $3.27^{\circ}, 5.66^{\circ}$, and $6.52^{\circ}$. The relative momentum spectra were formed for the $p-p$, $p-d, p-\alpha, d-\alpha$, and $\alpha-\alpha$ interactions from the coincidences. Correlation functions were formed for these five interactions by dividing a relative momentum spectrum by a reference spectrum. The reference spectrum was obtained by mixing 


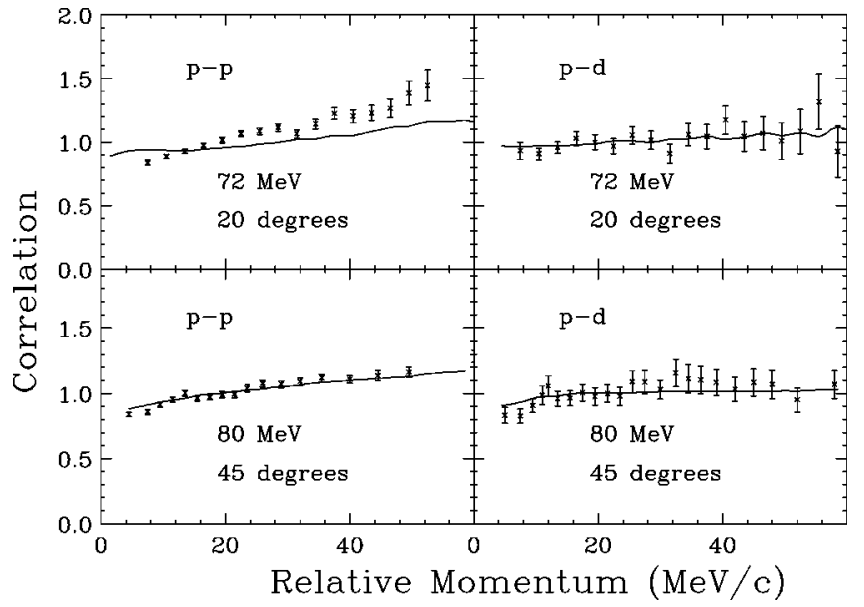

FIG. 1. Comparison of results from forward and backward scattering angles for $p-p$ and $p-d$. The beam energy and laboratory detection angle are indicated in each panel. The error bars are statistical but do include an estimate of the error associated with the formation of the reference spectrum. The solid lines are the results of Coulomb trajectory calculations assuming emission from an equilibrated compound nucleus.

events and the total counts in the reference spectra were taken to be the same as the real relative momentum spectra. Only events that involved the same opening angle and the same two LCP were mixed.

\section{RESULTS AND DISCUSSION}

The measured correlation functions are shown in Fig. 1 for the $p-p$ and $p-d$ exit channel. The $\alpha-\alpha, d-\alpha$, and $p$ - $\alpha$ results are given in Fig. 2. Also shown in these figures are the measured correlation functions for a similar energy (80 $\mathrm{MeV})$ but obtained at a more backward angle $\left(\theta_{\mathrm{lab}}=45^{\circ}\right)$ [2]. The minimum opening angle for the more backwardangle measurement was slightly larger $\left(4.62^{\circ}\right)$ than in this work, but the range of opening angles included in the data is comparable.

The general features of the data are as expected. In Fig. 1 one sees that the correlation functions are relatively featureless. The positive correlation due to diprotons at a relative momentum of $\approx 20 \mathrm{MeV} / c$, often seen at higher energies, is absent since this system is extended in size and long lived. However, there are downward trends to anticorrelation at the smallest values of the relative momentum. (Compared to the shape of a $p-p$ correlation function measured at higher energies, the correlation function displayed may not exhibit a "normal" shape. At very low energies, the limited energy range of the emitted protons and the event-mixing technique for the construction of the reference spectrum result in a correlation function that is not asymptotically flat at the higher values of relative momentum. This was also observed in Ref. [2].) In Fig. 2, one sees the expected peak at $18 \mathrm{MeV} / c$ in the $\alpha-\alpha$ correlation function corresponding to ${ }^{8} \mathrm{Be}$ breakup and the expected peak at $40 \mathrm{MeV} / c$ in the $d-\alpha$ correlation function from ${ }^{6} \mathrm{Li}^{*}$ production. The remaining $p-\alpha$ correlation function is relatively featureless because the width of the resonance in ${ }^{5} \mathrm{He}$ is very broad. (The small positive correlation seen near $20 \mathrm{MeV} / \mathrm{c}$ is due to the breakup of ${ }^{9} \mathrm{~B}$.) The forward angle results look very similar to the backward angle results.

The particular shape of any given correlation function is due to the interplay of the repulsive long-ranged Coulomb force, the short-ranged attractive nuclear force, and the breakup of any unstable nuclei. At lower energies, the characteristic large space-time extent of the sources results in correlation functions which are not strongly affected by the nuclear force and so do not have the strong positive correlation peak seen in some higher-energy experiments. As the space-time extent of the emitting system becomes smaller, the depth of the anticorrelation becomes deeper and the positive correlation begins to become apparent and grows. In this particular experiment, the $p-p$ and $p$ - $d$ correlations show a slight anticorrelation at the lowest values of relative momentum due to the Coulomb force. The other correlation functions show a significant positive correlation, not because the source is small (which would be inconsistent with the $p-p$ and $p$ - $d$ results), but because there are unstable nuclei which decay with definite kinematics. When the correlations are not strong, as for $p-p$ and $p$ - $d$, the particular shapes of the correlation functions may not have the expected asymptotic value of unity at large relative momentum because of the way the background relative momentum spectrum is formed. (In this analysis the area of the foreground and background spectra are fixed to be the same.) The surest way to correctly interpret the functions in these cases is to compare the measured correlation functions with modeled correlation functions constructed exactly in the same way as the data.

Thus, the simplest interpretation of these correlation functions indicates a source with an extended space-time extent. Given the beam energy, size of the compound nucleus, and excitation energy of the compound nucleus, one would expect such a large space-time extent.

However, a more detailed comparison between the forward angle and backward angle data for the $p$ - $p$ case shows an interesting feature. The shape in this case is significantly different from the other functions and indicates that the observed $p$ - $p$ pairs came from a source with a smaller average space-time size. Differences in the correlation functions shown in Fig. 2 can be understood in terms of differences in the production rate of ${ }^{8} \mathrm{Be}$ and ${ }^{6} \mathrm{Li}^{*}$ at different angles and energies [4]. It is more difficult to explain why the forwardangle $p-p$ correlation function should indicate different source properties.

The results were also modeled (solid lines in Fig. 1) to check that the observed differences did not arise from simple kinematic effects or the specifics of the detector geometry. With a statistical model code (MODGAN [5]) the properties of the particle emission (energy distributions at each decay step, decay probabilities, lifetime of each step, etc.) were determined. This information served as input to a Monte Carlo Coulomb trajectory calculation [6] with two important features. First the specific detector geometry was carefully reproduced. Second, the process of forming the reference spectrum for the model results was the same as that for the measured data. In the past, when this approach has been applied to low-energy correlation results the data have been 


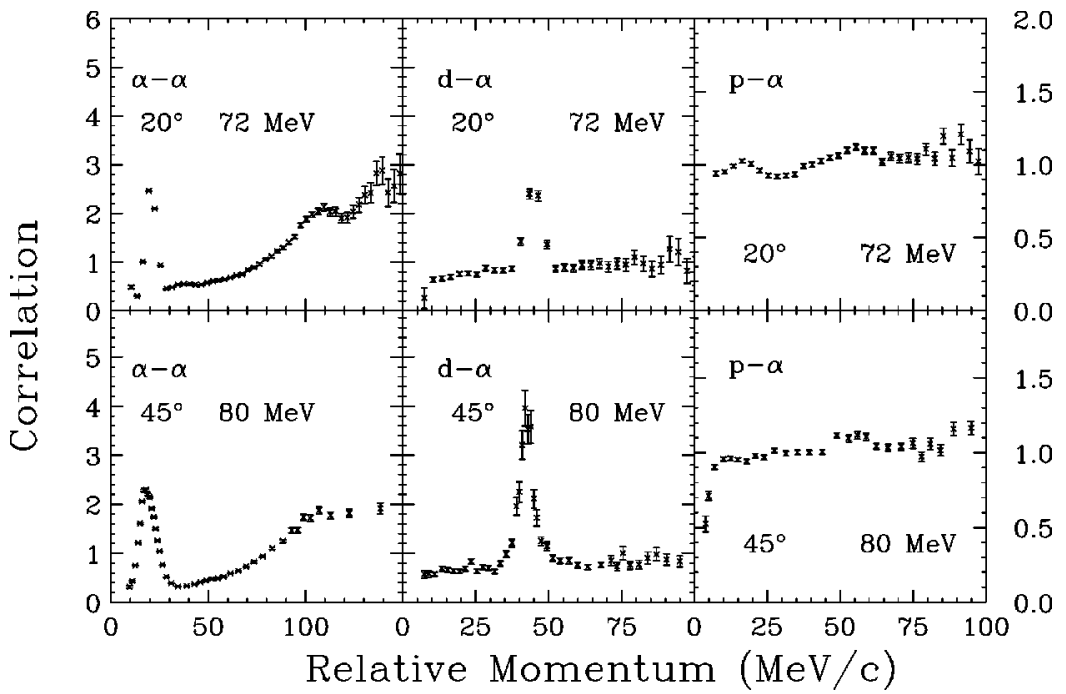

FIG. 2. Comparison of results from forward and backward scattering angles for $p-\alpha, d-\alpha$, and $\alpha-\alpha$. The beam energy and laboratory detection angle are indicated in each panel. The error bars are statistical but do include an estimate of the error associated with the formation of the reference spectrum.

well reproduced $[2,4,7]$. The forward-angle $p-p$ measurement deviates from the prediction significantly and this indicates the need for additional study.

The specific shape of the forward-angle $p-p$ correlation is not the standard one observed at higher energies where the correlation is flat at high values of relative momentum and exhibits a pronounced dip, due to Coulomb repulsion, at the lowest values of relative momentum. In this case, the specific shape of the forward-angle $p-p$ correlation function is determined by the space-time properties of the source but is also influenced by the limited range of proton momenta (this is a low-energy measurement) and the event-mixing algorithm. However, since the model results are treated the same way as the data, comparisons with the calculations are instructive and valid.

When simply comparing the four correlation functions, the downward trend at lower values of relative momentum of the forward-angle $p$ - $p$ data seems more pronounced. In addition, there is good agreement between the data and model calculations in three cases, but one sees a pronounced difference between the data and a model calculation for the forward $p-p$ result. This implies that the detected protons are originating from a source (or sources) that is different from that applicable to the other three correlation functions.

Prior work suggested that the wide variety of $p-p$ correlation results arose from a variety of reaction mechanisms which have different angular distributions and different space-time sizes [1]. One might have expected that the the current correlation results, at a beam energy of only $72 \mathrm{MeV}$, would be determined by the dominant mode of evaporation from a single equilibrated source. However, it is surprising that other processes could have a large enough cross section and multiplicity compared to evaporation from an equilibrated system to significantly affect the correlation function at this low energy. Still, even though the deviations from unity in the correlation functions are small at both forward and backward angles, the effect observed in the forwardangle $p-p$ correlation function is significant. Also, the corre- lation functions involving composite particles, especially $p-d$, seem unaffected. This suggests that the nonstatistical emission process manifests itself preferentially with protons rather than composite particles (such as $d$ ).

There is definitely something at work which causes the correlation functions for $p-p$ at more forward angles to indicate emission from a source with a smaller space-time extent. (Assuming emission from a compound nucleus, this would mean a shorter lifetime.) Obviously, this single measurement can not articulate the detailed interplay of mechanisms responsible for the observations. However, it does seem that the story told by correlation functions and the inferences drawn from such measurements must be interpreted with care.

\section{CONCLUSIONS}

The large number of correlation measurements done to date have demonstrated that the space-time size of the sources of LCP's affect the measured correlation function. The past low energy measurements (at more backward angles) of $p-p$ and $p-d$ correlation functions have been well reproduced by calculations based on the statistical model $[2,4,7]$ and the model is expected to apply to this latest measurement. This newest measurement indicates that, in addition to an energy dependence, an angular dependence has been observed for the $p$ - $p$ channel where none was expected. An explanation of the observation is that correlation functions are also sensitive to the presence of multiple reaction mechanisms and that, even at the low energy of this work, a source of LCP other than statistical emission from an equilibrated compound nucleus, is observed.

\section{ACKNOWLEDGMENTS}

This work was supported by the National Science Foundation under Grant Nos. PHY98-70262 (Hope College), PHY95-15517 (Hope College REU program), and PHY9402761 (Notre Dame). 
[1] P. A. DeYoung et al., Phys. Rev. C 56, 244 (1997); see also references therein.

[2] P. A. DeYoung et al., Phys. Rev. C 41, R1885 (1990).

[3] J. Alarja et al., Nucl. Instrum. Methods Phys. Res. A 242, 352 (1986).

[4] P. A. DeYoung, N. N. Ajitanand, J. M. Alexander, V. Datar, C. J. Gelderloos, G. Gilfoyle, M. S. Gordon, R. L. McGrath, G. F. Peaslee, and J. Sarafa, Phys. Rev. C 52, 3488 (1995).

[5] N. N. Ajitanand and J. M. Alexander, Nucl. Instrum. Methods
Phys. Res. A 376, 213 (1996).

[6] A. Elmaani, N. N. Ajitanand, T. Ethvignot, and J. M. Alexander, Nucl. Instrum. Methods Phys. Res. A 313, 401 (1992).

[7] P. A. DeYoung, M. S. Gordon, X. Q. Lu, R. L. McGrath, J. M. Alexander, D. M. de Castro Rizzo, and L. C. Vaz, Phys. Rev. C 39, 128 (1989); M. S. Gordon, R. L. McGrath, J. M. Alexander, P. A. DeYoung, X. Q. Lu, D. M. de Castro Rizzo, and G. P. Gilfoyle, ibid. 46, R1 (1992); R. A. Kryger et al., ibid. 46, 1887 (1992). 